\title{
Virtualization in anesthesiology. Projection of Anesthesiology as virtual environment for robotic virtual machine. Future of anesthetics in robotic surgery
}

\author{
Vinita Shukla* \\ Aievol Consulting Limited, London, TW3 2PA, UK
}

As a part of multi-disciplinary research anesthesiology is key in modern day surgery. This paper is emphasis on improvement metrics in anesthesiology by the use of Robotic Virtual Machine (RVM) in Virtual Environment (VE). Let the author take the opportunity to discuss what RVM can bring to the table. Time immemorial anesthetics have been involved in precision surgery where is also included RVM. Taking twenty years stride the author rests assured job loss can only be news only of the past and not a botheration in the current scenario. This is to implement and bring live the proverb complementary is certainly better than supplementary. The RVM is extension of human capability. The implementation of such can have happen by understanding VE as anesthesiology. Anesthetics are a substance that induces insensitivity to pain and anesthesiology is the study of anesthetics. Imagine a surgery without administering anesthetics to the patients. It is complete chaos and disaster. Thereby, this important stream that keeps the world of surgery going is worthy a glimpse and deep research with overview of what is the pinnacle of anesthetics in robotic surgery. The job and job prospect of RVM is quite similar to high artificial intelligence quotient and shall be seen in the future chapter. Thus, by the essence of philosophy of artificial intelligence and the philosophy of life, RVM is a human by practice and prospects. The essence as such cannot be mitigated by human. This brings us to the fact RVM is complement to our system and not supplements. It would be impressive to have RVMs as human extension and not create job loss. This system has been smartened by RVM without engaging in insecurities creation or spread. We are working on Virtualization of robotic system thereby there are already work on re-virtualization and repeat of several other degrees of achievement such as re-extension. The capability working as extension is not only key but also RVM does not miss the human essence. This successful reporting is important for successful business as the demarcation of capabilities is fact of life and not virtual anymore. The virtual reality is along VE has improved the performance of virtual environment in terms of quality and quantification and also performance of RVM in VE is noteworthy and performance that goes above all. This paper is quite not in agreement with the rest of the clan that have say jobs shall be lost. Thereby the innovation that walks in is welcome and desirous. Metrics of performance of RVM increases with the inclusion of anesthetics as the virtual environment. Even the most experienced surgeon will have say that inclusion of big software has not only improved the mechanical handling but also saved the big data from being thrown in the data lake. In the coming sections will be discussed how anesthesia can helps patients in recovery from pain, recovery with pain therapy and robotics aspect. What-if the machine gets faster than human expertise, will it not essential be to train RVM with human essence.
RVM as defined is robotic virtual machine that has software replace every hardware. Virtual environment is the playground of RVM. All in all, it is the study of RVM in VE. The RVM being the equipment even though it is estimated for such replacement in a century, it still is a brighter prospect and prosperous for most of researchers studying philosophy of Artificial Intelligence. The equipment and supplies would soon be replacement by RVM as an outcome of hardware replaced with software. The study in coming sections will deliver how metrics like reliability and scalability have improved. Also, we shall see the scope of RVM as innovator and as a process on the whole. There with virtualization is development of RVM to build provision for accurate anesthesia therapy. The precision of RVM in robotic surgery bearing in mind different locations simultaneous can be handled by a big software [1]. Having understood what RVM, VE, Artificial intelligence, replacement of equipment supplies, metric definition, coming sections define how metrics get better to acquire status of renewable energy and renewable resource. As human can have transition in energy for this aspect to be comparable to intelligence and emotional training of RVM, so that RVM can be extension of human capability. Also, as human, transition and renew their spirit so does anesthesia renew human to awaken state after a deep sleep. This similarity is as remarkable as philosophy of artificial intelligence RVM has built a similar or even better, a better intelligence and emotional capability like human thereby works successfully as their extension capability.

Concept of renewable energy in Anesthesiology- With the above stated explanation renewable resource is defined as a form of resource that can transition still define its usefulness and utility. RVM is outcome of virtualization is renewable resource. Some of its example is Virtualization. The outcome of its vast nature is no data is thrown in the data lake [2]. At this point of time philosophy of artificial intelligence is to determine if the system is intelligent and emotional like a human we determine if the outcome point 1 stated in figure 1 that follows now is desirous. The answer to it being yes is the start of generation of renewable energy but where does Anesthetics fall in the clear picture. Without much ado it has been explained it is anesthesia patient care

${ }^{\star}$ Correspondence to: Vinita Shukla, Aievol Consulting Limited, London, TW3 2PA, UK, E-mail: vinitashukla35@gmail.com

Key words: virtual environment, robotic virtual machine, virtualization and anesthetics, anesthesiology, metrics

Received: February 08, 2019; Accepted: March 08, 2019; Published: March 13, 2019 


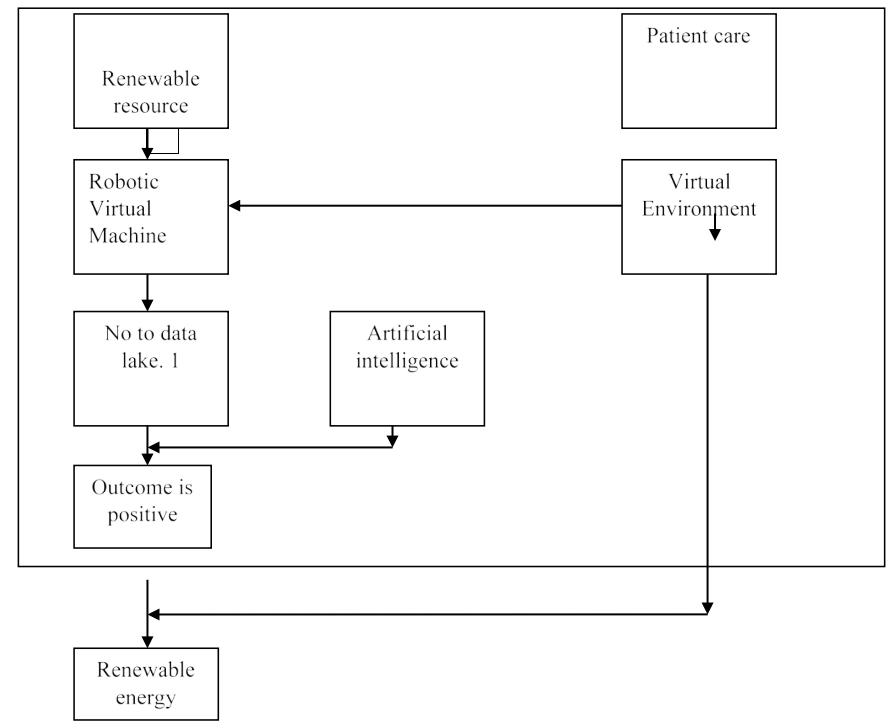

Figure 1. Illustration of similarity and outcome outcome that is the virtual environment [3]. Anesthesiology in surgical procedures, time immemorial, has paved its way to virtual reality and real. Renewable energy is energy that can transition from one useful form to the other. The AI quotient of RVM has improved [4].

\section{Conclusion}

Thus, the metrics like reliability, scalability and cost effectiveness have improved. The illustrious AI quotient also gets better is good news for data scientist as no more will the outcome be declared redundant as in the past.

\section{References}

1. Vinita Shukla (2018) Recognition of virtual environment VE by robot virtual machine RVM. JETIER 5: 115-116.

2. Vinita Shukla (2018) Robotic virtual machine RVM. Robotic virtualisation future of RVM without having to lose the pieces like a physical robot, the new era and innovation. JETIER 5: 673-677.

3. Vinita Shukla (2018) Robotic evolution and history. JETIER 5: 212-218.

4. Holland SW (1980) A vision-controlled robot for part transfer, (Industrial Robots) vol 2 second edition, society of Automative Engineering Inc.

Copyright: (C2019 Shukla V. This is an open-access article distributed under the terms of the Creative Commons Attribution License, which permits unrestricted use, distribution, and reproduction in any medium, provided the original author and source are credited. 